\title{
COVID-19: importância das novas tecnologias para a prática de atividades físicas como estratégia de saúde pública
}

\author{
COVID-19: the importance of new technologies \\ for physical activity as a public health strategy
}

\author{
COVID-19: importancia de las nuevas tecnologías \\ para la práctica de actividades físicas como \\ estrategia de salud pública
}

\author{
Breno Augusto Bormann de Souza Filho 1,2 \\ Érika Fernandes Tritany ${ }^{3}$
}

doi: $10.1590 / 0102-311 \times 00054420$
A pandemia relacionada ao novo coronavírus (SARS-CoV-2), causador da COVID-19 1, vem impactando negativamente na saúde da população e na economia mundial 2. Dados publicados em 28 de abril de 2020 registram a doença em 213 países, com aproximadamente 2.954 .222 casos confirmados, correspondendo a mais de 202.597 óbitos no mundo ${ }^{3}$, sendo os idosos o grupo mais vulnerável. É importante ressaltar que a subnotificação dos dados é uma questão pertinente a todos os países que enfrentam a doença, portanto, o quantitativo apresentado pela Organização Mundial da Saúde (OMS) e países está sub-representado. No Brasil, após notificação do primeiro caso, em fevereiro de 2020, observou-se um crescente aumento no número de casos confirmados, suspeitos e óbitos relacionados a COVID-194.

Dada a inexistência de profilaxia, tratamento específico e sua alta taxa de transmissibilidade, o Brasil decretou Emergência de Saúde Pública de Importância Nacional (ESPIN) 5,6 e, seguindo recomendações e experiências internacionais, adotou o isolamento social como a principal medida de prevenção e controle da doença 7 . Foram, assim, suspensas grande parte das atividades laborais, físicas e recreativas 7,8,9,10,11,12, sendo proibida a permanência em praias e praças públicas, estando sujeitos os cidadãos à punição penal por desobediência 13,14, alterando ainda mais a dinâmica social.

Importantes esforços da vigilância e serviços de saúde estão, principalmente, focados na contenção da epidemia e promoção de estratégias que viabilizem a ação coordenada e oportuna do sistema de saúde, o qual já está sobrecarregado. Entretanto, as doenças crônicas e comorbidades preexistentes continuam a ser um problema de grande magnitude - principalmente para idosos, grupo mais afetado pelo vírus - e que requerem ações de tratamento, controle e monitoramento contínuos, associados ao estilo de vida 15 , além do impacto econômico que a inatividade física da população pode gerar aos sistemas de saúde e indivíduos 16.

Apesar de ser uma medida estratégica para a contenção de casos, o isolamento social pode trazer consequências negativas não intencionais, impactando no aumento do sedentarismo e inatividade física da população, considerada por muitos autores, por si só, como uma pandemia 17. A estada prolongada limitada à residência pode promover a diminuição da interação social e maiores períodos de imobilidade (sentado ou deitado) direta ou indiretamente relacionados à maior utilização de equipamentos virtuais como TV, computadores, celulares ou outros 18. Podem também ocorrer alterações no nível motivacional para a prática de atividades físicas, pois estas, muitas vezes, são associadas à
1 Escola Nacional de Saúde Pública Sergio Arouca, Fundação Oswaldo Cruz, Rio de Janeiro, Brasil. 2 Academia Paralímpica Brasileira, Rio de Janeiro, Brasil.

3 Universidade Federal do Rio de Janeiro, Rio de Janeiro, Brasil.

Correspondência B. A. B. Souza Filho Av. Prado Júnior 330, apto. 406, Rio de Janeiro, $R J$ 22011-040, Brasil. brenobormann@hotmail.com 
necessidade de estrutura física, equipamentos complexos e de alto custo; locais específicos como parques, praças, clubes; e/ou suporte profissional presencial 19.

Entretanto, os benefícios da atividade física estão relacionados à sua prática e não exclusivamente a esses fatores. Sua realização apresenta efeitos imediatos e a longo prazo na saúde 20: além de diminuir efeitos nocivos relacionados a grandes períodos de imobilidade, favorece o controle das doenças crônicas e comorbidades a elas associadas 15, e melhora a resposta imunológica a infecções 21, o que pode impactar na gravidade dos sintomas e desfecho do quadro clínico de pacientes com COVID-19 22 ou outras doenças transmissíveis; promove ganhos funcionais globais, qualidade de vida e favorece a diminuição do estresse e ansiedade, sintomas comuns em situações de crise social 23.

Por sua vez, a inatividade física é considerada fator de risco chave para doenças crônicas não transmissíveis, como as cardiovasculares, cânceres e diabetes 15,20, principais comorbidades associadas a quadros complexos da síndrome respiratória aguda grave (SRAG) por COVID-19 22 e principais causas de morte no mundo 24 . Assim, o desenvolvimento e adoção de metodologias alternativas que favoreçam a autonomia das pessoas e promovam a realização e manutenção da atividade física são de extrema importância para a promoção da saúde, prevenção e controle de doenças 25 . Nesse sentido, o estímulo à realização de atividades físicas domiciliares apresenta-se como uma proposta importante e viável, sobretudo para grupos vulneráveis e/ou durante períodos emergenciais de restrição social, como vivenciados pela pandemia de COVID-19.

A despeito dessas questões, até o presente momento o Ministério da Saúde e governos estaduais e municipais não apresentaram, por meio dos dispositivos de ação para o enfrentamento da COVID-19, propostas e recomendações para a população e estabelecimentos sobre a importância da realização de atividade física durante períodos de reclusão social. O Conselho Federal de Educação Física (CONFEF), por sua vez, recomendou aos profissionais de educação física o estímulo e orientação ao beneficiário a permanecer fisicamente ativo, inclusive na residência, respeitando eventuais contraindicações específicas 26 . Porém, essas recomendações não foram estendidas aos estabelecimentos.

Haja vista a incerteza sobre quando poderá ser seguro deixar o isolamento, a situação de inatividade física pode se agravar. A importância de recomendações por parte dos órgãos reguladores e instâncias governamentais está centrada no potencial de influenciar estabelecimentos e profissionais à adoção de práticas. Além disso, têm um importante papel em conferir legitimidade e regulamentar práticas inovadoras, respaldando o exercício profissional e encorajando profissionais a ampliar suas práticas.

É importante que o Ministério da Saúde e Secretarias de Saúde tenham planos intersetoriais para a promoção de atividade física, fortalecendo o combate à inatividade física, e a promoção de estilos de vidas saudáveis como prevenção e tratamento de doenças crônicas ${ }^{17}$. Nesse sentido, é urgente a emissão de recomendações oficiais gerais à população com relação à prática de atividade física, em particular as domiciliares, no período de isolamento social, bem como a profissionais e estabelecimentos.

Programas de atividades físicas domiciliares são considerados eficazes, seguros e de baixo custo, e quando realizados de forma direcionada, de acordo com a especificidade de cada indivíduo, promovem ganhos nos componentes da aptidão física relacionados à saúde e habilidades, impactando positivamente na funcionalidade global e qualidade de vida destes 27,28. Apresentam, assim, uma alternativa promissora e eficaz para o aumento e manutenção dos níveis de atividade física da população e de resposta imunológica ao SARS-CoV-2, a ser adotada como política pública de saúde.

Além de importantes estratégias gerais, como as recomendadas pela OMS para a manutenção do estilo de vida ativo - como a realização de, pelo menos, 150 minutos semanais de atividade física moderada para adultos 24 - alguns indivíduos podem necessitar de programas específicos, adequados às suas condições físicas e psicossociais, o que pode favorecer a adesão e atenuar riscos relacionados a comorbidades preexistentes 29 .

Sempre que possível, devem ser considerados os componentes para a prescrição de atividade física - frequência, intensidade, tempo, tipo, volume, e progressão da atividade - e verificar os possíveis efeitos que cada componente pode exercer e sofrer em relação às especificidades dos indivíduos e grupos, como faixa etária, progressão de doenças, tratamentos específicos, sintomas, situação de vulnerabilidade social, status laboral, fragilidade emocional, risco de quedas, orientação religiosa, entre outros 29 . 
Assim, é apresentada uma proposta de abordagem holística que relacione a prática de atividade física não apenas ao status funcional e à autoavaliação subjetiva, pelos praticantes, de seu nível de aptidão física para a realização do programa proposto, mas apresente ações factíveis dentro das preferências e possibilidades de cada pessoa.

Quando possível, oportunizar uma variedade de modalidades como dança, exercícios resistidos, ioga, jogos e outros; e compreender fatores que podem influenciar no declínio funcional global e condições que levem a incapacidades 30 , bem como aspectos físicos, sociais, emocionais e espirituais que possam impactar na prática de atividade física, auxilia na adesão e adequação da proposta ao grupo ao qual é direcionado.

Assim, a criação de canais de comunicação remotos entre profissionais e usuários/clientes pode possibilitar a adequação, quando necessário, do programa ofertado e avaliar seus possíveis impactos à saúde. Essas ações podem ser adotadas por profissionais de educação física liberais, como personal trainers, bem como pelos estabelecimentos de saúde e atividade física, públicos e privados. Os profissionais de educação física vinculados às unidades de atenção primária, por exemplo, podem desenvolver programas de atividade física - baseados em materiais instrucionais e comunicação remota - prescrições e avaliações, de acordo com grupos, favorecendo a continuidade do cuidado e a manutenção do vínculo.

Essas recomendações são válidas tanto para períodos de isolamento social, imposto pela situação de epidemias e pandemias, quanto para períodos de "normalidade", direcionadas a grupos vulneráveis ou com fragilidades que dificultam a saída de casa, ou por preferências dos indivíduos.

Reforçamos que a prática de atividades físicas, inclusive em domicílio, deve ser uma política de saúde pública amplamente divulgada e difundida que se adeque às novas dinâmicas sociais e busque incentivar a autonomia e independência das pessoas, fortalecendo o autocuidado apoiado.

É importante levar em consideração que períodos de isolamento social, normalmente, não são experienciados a longo prazo, entretanto, seus impactos podem ser vivenciados por toda uma vida. Portanto, requerem ações coordenadas e pautadas em uma concepção de saúde ampliada, atenta às diferenças entre grupos e iniquidades em saúde. Nesse sentido, torna-se emergencial a recomendação de estratégias que auxiliem as pessoas a manterem-se ou tornarem-se fisicamente ativas em domicílio, fomentando a autonomia destas no gerenciamento e tomada de decisões sobre suas vidas.

\section{Colaboradores}

Ambos os autores participaram da concepção, projeto, redação, revisão crítica e aprovação da versão final.

\section{Informacões adicionais}

ORCID: Breno Augusto Bormann de Souza Filho (0000-0002-1700-8688); Érika Fernandes Tritany (0000-0002-7099-4800).

\section{Referências}

1. Andersen KG, Rambaut A, Lipkin WI, Holmes EC, Garry RF. The proximal origin of SARSCoV-2. Nat Med 2020; 26:450-2.

2. Ayittey FK, Ayittey MK, Chiwero NB, Kamasah JS, Dzuvor C. Economic impacts of Wuhan 2019-nCoV on China and the world. J Med Virol 2020; 92:473-5.

3. World Health Organization. Novel coronavirus (COVID-19) situation. https://experience.arc gis.com/experience/685d0ace521648f8a5beee ee1b9125cd (acessado em 21/Mar/2020).

4. Ministério da Saúde. Coronavírus: o que você precisa saber e como prevenir o contágio. https://coronavirus.saude.gov.br/ (acessado em 28/Abr/2020). 
5. Brasil. Lei no 13.979, de 6 de fevereiro de 2020. Dispõe sobre as medidas para enfrentamento da emergência de saúde pública de importância internacional decorrente do coronavírus responsável pelo surto de 2019. Diário Oficial da União 2020; 7 fev.

6. Ministério da Saúde. Portaria no 188 , de 3 de fevereiro de 2020. Declara Emergência em Saúde Pública de importância Nacional (ESPIN) em decorrência da Infecção Humana pelo novo Coronavírus (2019-nCoV). Diário Oficial da União 2020; 4 fev.

7. Anderson RM, Heesterbeek H, Klinkenberg D, Hollingsworth TD. How will country-based mitigation measures influence the course of the COVID-19 epidemic? Lancet 2020; 395:931-4.

8. Monteiro N, Aquino V, Pacheco S, Scheneiders L. Saúde anuncia orientações para evitar a disseminação do coronavírus. Agência Saúde 2020; 13 mar. https://www.saude.gov.br/noti cias/agencia-saude/46540-saude-anuncia-o rientacoes-para-evitar-a-disseminacao-docoronavirus.

9. Rio de Janeiro. Decreto no 46.973, de 16 de março de 2020. Reconhece a situação de emergência na saúde pública do Estado do Rio de Janeiro em razão do contágio e adota medidas enfrentamento da propagação decorrente do novo coronavírus (COVID-19); e dá outras providências. Diário Oficial do Estado do Rio de Janeiro 2020; 18 mar.

10. São Paulo. Decreto no 64.862, de 13 de março de 2020. Dispõe sobre a adoção, no âmbito da Administração Pública direta e indireta, de medidas temporárias e emergenciais de prevenção de contágio pelo COVID-19 (Novo Coronavírus), bem como sobre recomendações no setor privado estadual. Diário Oficial do Estado de São Paulo 2020; 14 mar.

11. São Paulo. Decreto no 64.864, de 16 de março de 2020. Dispõe sobre a adoção de medidas adicionais, de caráter temporário e emergencial, de prevenção de contágio pelo COVID-19 (Novo Coronavírus), e dá providências correlatas. Diário Oficial do Estado de São Paulo 2020; 17 mar.

12. São Paulo. Decreto no 64.865, de 18 de março de 2020. Acrescenta dispositivo ao Decreto no 64.862, de 13 de março de 2020, que dispõe sobre a adoção, no âmbito da Administração Pública direta e indireta, de medidas temporárias e emergenciais de prevenção de contágio pelo COVID-19 (Novo Coronavírus), bem como sobre recomendações no setor privado estadual. Diário Oficial do Estado de São Paulo 2020; 19 mar.
13. Niterói. Decreto no 13.513 , de 19 de março de 2020. Dispõe sobre o fechamento de bares, restaurantes, shoppings centers, centros comerciais, clubes e quiosques de alimentação, bem como sobre a proibição de permanência em praias e praças e fechamento de acesso às praias da região oceânica como forma de evitar a aglomeração de pessoas e evitar o crescimento dos casos de coronavírus em niterói e dá outras providências. Diário Oficial 2020; 19 mar.

14. Rio de Janeiro. Decreto no 46.980, de 19 de março de 2020. Atualiza as medidas de enfrentamento da propagação decorrente do novo coronavírus (COVID-19) em decorrência da situação de emergência em saúde e dá outras providências. Diário Oficial do Estado do Rio de Janeiro 2020; 19 mar.

15. Ekelund U, Steene-Johannessen J, Brown WJ, Fagerland MW, Owen N, Powell KE, et al. Does physical activity attenuate, or even eliminate, the detrimental association of sitting time with mortality? A harmonised meta-analysis of data from more than 1 million men and women. Lancet 2016; 388:1302-10.

16. Ding D, Lawson KD, Kolbe-Alexander TL, Finkelstein EA, Katzmarzyk PT, van Mechelen W, et al. The economic burden of physical inactivity: a global analysis of major non-communicable diseases. Lancet 2016; 388:1311-24.

17. Reis RS, Salvo D, Ogilvie D, Lambert EV, Goenka S, Brownson RC. Scaling up physical activity interventions worldwide: stepping up to larger and smarter approaches to get people moving. Lancet 2016; 388:1337-48.

18. Brazendale K, Beets MW, Weaver RG, Pate RR, Turner-McGrievy GM, Kaczynski AT, et al. Understanding differences between summer vs. school obesogenic behaviors of children: the structured days hypothesis. Int J Behav Nutr Phys Act 2017; 14:100.

19. Marcus B, Forsyth L. Motivating people to be physically active. 2nd Ed. Champaign: Human Kinetics; 2009. (Physical Activity Intervention Series).

20. Lee I-M, Shiroma EJ, Lobelo F, Puska P, Blair SN, Katzmarzyk PT. Effect of physical inactivity on major non-communicable diseases worldwide: an analysis of burden of disease and life expectancy. Lancet 2012; 380:219-29.

21. Campbell JP, Turner JE. Debunking the myth of exercise-induced immune suppression: redefining the impact of exercise on immunological health across the lifespan. Front Immunol 2018; 9:648. 
22. Zhou F, Yu T, Du R, Fan G, Liu Y, Liu Z, et al. Clinical course and risk factors for mortality of adult inpatients with COVID-19 in Wuhan, China: a retrospective cohort study. Lancet 2020; 395:1054-62.

23. Bushman BA; American College of Sports Medicine, organizadores. ACSM's complete guide to fitness \& health. 2nd Ed. Champaign: Human Kinetics; 2017.

24. World Health Organization. More active people for a healthier world: Global Action Plan on Physical Activity 2018-2030. Geneva: World Health Organization; 2018.

25. Wang G, Zhang Y, Zhao J, Zhang J, Jiang F. Mitigate the effects of home confinement on children during the COVID-19 outbreak. Lancet 2020; 395:945-7.

26. Conselho Federal de Educação Física. Recomendações do CONFEF aos profissionais de educação física no contexto da COVID-19. https:// www.confef.org.br/confef/comunicacao/noti cias/1475 (acessado em 20/Mar/2020).

27. Matias GHL, Guerra ACCG, Souza Filho BAB, Lima JTO, Carmo CN, Mattos IE. Repetibilidade e reprodutibilidade de um manual de exercícios físicos domiciliares. Fisioter Pesqui 2018; 25:209-16.
28. Souza Filho BAB, Silva Júnior JR, Smethurst WS, Santos DC, Carmo CN, Mattos IE, et al. Efeito de 12 semanas de exercício físico domiciliar na aptidão física de idosas com câncer de mama em hormonioterapia. Acta Fisiátr 2019; 26(1). https://www.revistas.usp.br/actafisiatri $\mathrm{ca} /$ article/view/163420.

29. American College of Sports Medicine; Riebe D, Ehrman JK, Liguori G, Magal M, organizadores. ACSM's guidelines for exercise testing and prescription. 10th Ed. Philadelphia: Wolters Kluwer; 2018.

30. Rikli RE, Jones CJ. Senior fitness test manual. 2nd Ed. Champaign: Human Kinetics; 2012.

Recebido em 21/Mar/2020

Versão final reapresentada em 29/Abr/2020

Aprovado em 30/Abr/2020 